\title{
SMOOTHING CURVES BY REFLEXIVE SHEAVES
}

\author{
GIORGIO BOLONDI
}

(Communicated by Walter Littman)

\begin{abstract}
We give smoothing theorems for singular projective curves, by using curvilinear sheaves. This allows us to smooth reducible curves obtained by liaison addition.
\end{abstract}

1. Introduction and preliminaries. Several authors (classical and modern) used reducible curves in order to get, by smoothing, smooth curves. For example, [HII, HH, HI2, BE, SER] and others developed for this aim smoothing techniques (see $[\mathbf{H H}]$ for a general insight on this problem).

In this paper we show a way of using reflexive sheaves of a particular kind called "curvilinear" in order to get smoothing results; our techniques allow us to avoid the use of the normal bundle, which is sometimes difficult to handle (and which is needed in other known smoothing techniques). They seem to work very well in smoothing liaison addition; they have been already used in constructing smooth minimal models of curves in fixed liaison classes [BM].

In some way, these techniques go in the opposite direction than the papers quoted above, since we need very special curves, whilst they need curves with a low speciality index. But for several applications (for instance in smoothing liaison addition and for $r$-regular curves, in the sense of [GLP]) this is a natural situation.

We conclude by giving several applications and examples in the first cases.

We are concerned with nodal curves in $\mathrm{P}_{k}^{3}$, where $k$ is an algebraically closed field, that is to say reduced curves with only ordinary double points. We refer to $[\mathbf{H H}]$ for a general discussion of the definition of a smoothable curve.

DEFINITION 1.1. A rank-two reflexive sheaf $\mathcal{F}$ on $\mathrm{P}_{k}^{3}$ is said to be curvilinear if it has the following property: $\mathcal{F}(s)$ globally generated $\Rightarrow$ the zero set of a general section of $\mathcal{F}(s)$ is a smooth curve.

The central result about rank-two reflexive sheaves on $\mathrm{P}_{k}^{3}$ is contained in [HA]: there is a correspondence between pairs ( $₹$ sheaf, global section) and pairs $(Y$ curve, section of $\omega_{Y}\left(c_{1}(\mathcal{F})\right)$ ), where "curve" means a locally Cohen-Macaulay curve $Y$ generically locally complete intersection, and the section of $\omega_{Y}\left(c_{1}(\mathcal{F})\right)$ generates this sheaf except at finitely many points.

Hirschowitz [HI2] proved the following.

PROPOSITION 1.2. Let $Y$ be a curve and $f$ a section of $\omega_{Y}(4-p)$ which generates this sheaf in a neighborhood of the singular locus of $Y$. Then the sheaf associated to the pair $(Y, f)$ is curvilinear.

Received by the editors December 22, 1986.

1980 Mathematics Subject Classification (1985 Revision). Primary 14H45; Secondary 14 F05.

Key words and phrases. Curves, smoothing, reflexive sheaves. 
We recall something about "liaison addition". We call Hartshorne-Rao module of a curve $Y$ the module $M(Y)=\bigoplus_{n} H^{1}\left(\mathrm{P}_{k}^{3}, I_{Y}(n)\right)$. Liason addition is a procedure, introduced by Schwartau in [SCH], which allows us to get, starting from two curves $Y$ and $Z$, a new reducible curve $X$ whose Hartshorne-Rao module is $M(X)=$ $M(Y)(s) \oplus M(Z)(t)$, where $s$ and $t$ are positive integers. It works as follows: let $\Sigma_{s}$ be a surface a degree $s$ containing $Z$ and $\Sigma_{t}$ be a surface of degree $t$ containing $Y$, and let $C=\Sigma_{s} \cap \Sigma_{t}$. If $X=Y \cup Z \cup C$, then $M(X)=M(Y)(-s) \oplus M(Z)(-t)$.

\section{The general statement.}

THEOREM 2.1. Let $X \subset \mathrm{P}_{k}^{3}$ be a nodal curve (maybe reducible) such that there exists $\xi \in H^{0}\left(X, \omega_{X}(-t)\right)$ different from zero on the singularities of $X$, and $h^{1}\left(\mathrm{P}_{k}^{3}, I_{X}(t+3)\right)=0=h^{2}\left(\mathrm{P}_{k}^{3}, I_{X}(t+2)\right)$ for some $t \geq-2$. Then there exists a family of curves (to which $X$ belongs) parametrized by an open subset of $\mathrm{P}^{N}$ such that the general curve $Y$ is smooth and $h^{i}\left(\mathrm{P}_{k}^{3}, I_{X}(n)\right)=h^{i}\left(\mathrm{P}_{k}^{3}, I_{Y}(n)\right)$ for every $i$ and $n$.

PROOF. Let us suppose $t$ even, $t=2 p(p \geq-1)$. We can associate a reflexive sheaf $₹$ with $c_{1}(\mathcal{F})=0$ to the pair $(X, \xi)$ by means of the exact sequence

$$
0 \rightarrow \mathrm{O}_{\mathrm{P}^{3}}(-p-2) \rightarrow \mathcal{F} \rightarrow I_{X}\left(p_{2}\right) \rightarrow 0
$$

since $\xi \in H^{0}\left(X, \omega_{X}(-t)\right)=H^{0}\left(X, \omega_{X}(4-2(p+2))\right)$.

Thanks to Proposition $1.2, \mathcal{F}$ is curvilinear. From the long exact sequence associated to $(*)$, we get $h^{1}\left(\mathrm{P}_{k}^{3}, \mathcal{F}(p+1)\right)=h^{1}\left(\mathrm{P}_{k}^{3}, I_{X}(2 p+3)\right)=0$, and $0 \rightarrow$ $H^{2}\left(\mathrm{P}_{k}^{3}, \mathcal{F}(p)\right) \rightarrow H^{2}\left(\mathrm{P}_{k}^{3}, I_{X}(2 p+2)\right) \rightarrow H^{3}\left(\mathrm{P}_{k}^{3}, \mathrm{O}_{\mathrm{P}^{3}}(-2)\right)$; hence $h^{2}\left(\mathrm{P}_{k}^{3}, \mathcal{F}(p)\right)=$ $h^{2}\left(\mathrm{P}_{k}^{3}, I_{X}(2 p+2)\right)=0$.

On the other hand, by Serre's duality $[\mathbf{H A}], h^{3}\left(\mathrm{P}_{k}^{3}, \mathcal{F}(p-1)\right)=h^{0}\left(\mathrm{P}_{k}^{3}, \mathcal{F}(-p-3)\right)$, and this last group vanishes since, in the exact sequence

$$
0 \rightarrow H^{0}\left(\mathrm{P}_{k}^{3}, \mathrm{O}_{\mathrm{P}^{3}}(-2 p-5) \rightarrow H^{0}\left(\mathrm{P}_{k}^{3}, \mathcal{F}(-p-3)\right) \rightarrow H^{0}\left(\mathrm{P}_{k}^{3}, I_{X}(-1)\right),\right.
$$

we have the first and the third groups equal to zero.

So, we can apply to $\mp$ the lemma of Castelnuovo-Mumford: $F(p+2)$ is globally generated; therefore the zero set $Y$ of the general section of $\mathcal{F}(p+2)$ is smooth.

Remark that two sections $s$ and $s^{\prime}$ of $\mathcal{F}(p+2)$ have the same zero set if and only if $s=\lambda s^{\prime}$ for some $\lambda \in k^{*}$; therefore we have an injective morphism from $\mathrm{P}\left(H^{0}\left(\mathrm{P}_{k}^{3}, \mathcal{F}(p+2)\right)\right)$ to the Hilbert scheme of closed subscheme of $\mathrm{P}_{k}^{3}$. The general element of $\mathrm{P}\left(H^{0}\left(\mathrm{P}_{k}^{3}, \mathcal{F}(p+2)\right)\right)$ corresponds to a smooth curve, whilst a particular element corresponds to $X$.

The statement about the cohomology groups follows immediately from the exact sequences

$$
0 \rightarrow \mathrm{O}_{\mathrm{P}^{3}}(-p-2) \rightarrow \mathcal{I} \rightarrow I_{X}(p+2) \rightarrow 0
$$

and

$$
0 \rightarrow \mathrm{O}_{\mathrm{P}^{3}}(-p-2) \rightarrow \mathcal{F} \rightarrow I_{Y}(p+2) \rightarrow 0 .
$$

If $t$ is odd we can use a sheaf with $c_{1}=-1$.

3. Application to liaison addition. The previous general proposition has several applications; indeed, it works well in "smoothing" liaison addition (a procedure which by definition gives reducible curves). 
DEFINITION 3.1 (see [GLP]). A curve $X \subset \mathrm{P}^{3}$ is said to be $n$-regular if $h^{2}\left(\mathrm{P}_{k}^{3}, I_{X}(n-2)\right)=h^{1}\left(\mathrm{P}_{k}^{3}, I_{X}(n-1)\right)=0$. A curve $X$ is said to be strongly $n$-regular if it is $n$-regular and $h^{2}\left(\mathrm{P}_{k}^{3}, I_{X}(n-4)\right) \neq 0$.

If a curve is $n$-regular, then for every $p \geq n$ the sheaf $I_{X}(p)$ is globally generated, and $X$ is cut out in $\mathrm{P}^{3}$ by hypersurfaces of degree $n$. We can get strongly $n$-regular curves, for instance, from suitable sections of a reflexive sheaf with seminatural cohomology (see [HI2]). Moreover, strongly $n$-regularity is a common situation for curves minimal in their liaison class (see $[\mathbf{B M}]$ ).

THEOREM 3.2. Let $Y, Z \subset \mathrm{P}_{k}^{3}$ be smooth curves such that $Y$ is strongly sregular and $Z$ strongly $t$-regular. Then it is possible to "add by liaison" $Y$ and $Z$ (first moving them in $\mathrm{P}_{k}^{3}$ if necessary) by means of $\Sigma_{s} \supset Y$ and $\Sigma_{t} \supset Z$ in such a way that $X=Y \cup Z \cup\left(\Sigma_{s} \cap \Sigma_{t}\right)$ is smoothable.

Proof. We have

$$
\begin{aligned}
& 0 \neq h^{2}\left(\mathrm{P}_{k}^{3}, I_{Y}(s-4)\right)=h^{1}\left(Y, O_{Y}(s-4)\right)=h^{0}\left(Y, \omega_{Y}(4-s)\right), \\
& 0 \neq h^{2}\left(\mathrm{P}_{k}^{3}, I_{Z}(t-4)\right)=h^{1}\left(Z, O_{Z}(t-4)\right)=h^{0}\left(Z, \omega_{Z}(4-t)\right) .
\end{aligned}
$$

We fix nontrivial sections $\xi \in H^{0}\left(Y, \omega_{Y}(4-s)\right)$ and $\eta \in H^{0}\left(Z, \omega_{Z}(4-t)\right)$. $I_{Y}(s)$ and $I_{Z}(t)$ are globally generated (thanks to Castelnuovo-Mumford); therefore there exist a smooth surface $\Sigma_{s} \supset Y\left(\operatorname{deg} \Sigma_{s}=s\right)$ and another on $\Sigma_{t} \supset Z\left(\operatorname{deg} \Sigma_{t}=t\right)$; changing, if necessary, the position of $Y$ or $Z$ in $\mathrm{P}_{k}^{3}$, we can suppose that $Y \cap Z=\varnothing$ and that $C=\Sigma_{s} \cap \Sigma_{t}$ is smooth and cuts $Y$ and $Z$ transversally in points where $\xi$ and $\eta$ are different from zero.

The restriction morphism

$$
\rho: H^{0}\left(\mathrm{P}_{k}^{3}, \mathrm{O}_{\mathrm{P}^{3}}(t+s-4)\right) \rightarrow H^{0}\left(C, \mathrm{O}_{C}(t+s-4)\right)
$$

is surjective, since $C$ is a complete intersection. Let $X=Y \cup Z \cup C ; X$ is therefore the curve obtained by liaison addition from $Y$ and $Z$ by means of $\Sigma_{s}$ and $\Sigma_{t}$. The following restriction map is surjective also:

$$
\tau: H^{0}\left(X, O_{X}(t+s-4)\right) \rightarrow H^{0}\left(C, O_{C}(t+s-4)\right) .
$$

But this morphism $\tau$ is the dual map of the morphism

$$
\pi: H^{1}\left(C, \omega_{C}(4-s-t)\right) \rightarrow H^{1}\left(X, \omega_{X}(4-s-t)\right),
$$

which therefore turns out to be injective (see for instance [CO, Lemma 1.3]). There is an exact sequence

$$
0 \rightarrow \omega_{C} \rightarrow \omega_{X} \rightarrow \omega_{X \mid Y \cup Z} \rightarrow 0
$$

(see again [CO, lemma cit.]) from which we get a long exact sequence

$$
\begin{aligned}
H^{0}\left(X, \omega_{X}(4-s-t)\right) \stackrel{\delta}{\rightarrow} H^{0}\left(X, \omega_{X}(4-s-t)_{\mid Y \cup Z}\right) \\
\quad \rightarrow H^{1}\left(C, \omega_{C}(4-s-t)\right) \stackrel{\pi}{\rightarrow} H^{1}\left(X, \omega_{X}(4-s-t)\right)
\end{aligned}
$$

therefore the restriction map $\delta: H^{0}\left(X, \omega_{X}(4-s-t)\right) \rightarrow H^{0}\left(X, \omega_{X}(4-s-t)_{\mid Y \cup Z}\right)$ is surjective. But we have the following sheaf isomorphisms:

$$
\begin{aligned}
& \omega_{X}(4-s-t)_{\mid Y \cup Z} \cong \omega_{X}(4-s-t)_{\mid Y} \oplus \omega_{X}(4-s-t)_{\mid Z} \\
& \quad \cong\left[\omega_{Y}(4-s-t) \otimes O_{Y}(t)\right] \oplus\left[\omega_{Z}(4-s-t) \otimes O_{Z}(s)\right] \cong \omega_{Y}(4-s) \oplus \omega_{Z}(4-t) .
\end{aligned}
$$


Let $\varphi \in H^{0}\left(X, \omega_{X}(4-s-t)\right)$ be such that $\delta(\varphi)=\xi \oplus \eta \in \omega_{Y}(4-s) \oplus \omega_{Z}(4-t)$; $\varphi$ is a section of $\omega_{X}(4-s-t)$ which is different from zero on $\operatorname{Sing} X$.

Now we want to prove that

$$
h^{1}\left(\mathrm{P}_{k}^{3}, I_{X}(t+s-1)\right)=0=h^{2}\left(\mathrm{P}_{k}^{3}, I_{X}(t+s-2)\right) .
$$

In fact,

$$
H^{1}\left(\mathrm{P}_{k}^{3}, I_{X}(t+s-1)\right)=H^{1}\left(\mathrm{P}_{k}^{3}, I_{Y}(s-1)\right) \oplus H^{1}\left(\mathrm{P}_{k}^{3}, I_{Z}(t-1)\right)=0
$$

$[\mathbf{S C H}]$.

Moreover,

$$
O_{Z \cup Y}(-(Y \cup Z) \cap C) \cong O_{Y}(-Y \cap C) \oplus O_{Z}(-Z \cap C) \cong O_{Y}(-t) \oplus O_{Z}(-s)
$$

and the restriction morphism $H^{0}\left(X, O_{X}(t+s-2)\right) \rightarrow H^{0}\left(C, O_{C}(t+s-2)\right)$ is surjective. Therefore from the exact sequence

$$
0 \rightarrow O_{Z \cup Y}(-(Y \cup Z) \cap C) \rightarrow O_{X} \rightarrow O_{C} \rightarrow 0
$$

we get a long exact sequence

$$
\begin{aligned}
0 & \rightarrow H^{1}\left(Y, O_{Y}(s-2)\right) \oplus H^{1}\left(Z, O_{Z}(t-2)\right) \\
& \rightarrow H^{1}\left(X, O_{X}(t+s-2)\right) \rightarrow H^{1}\left(C, O_{C}(t+s-2)\right) \rightarrow 0
\end{aligned}
$$

Since it is

$$
H^{1}\left(Y, O_{Y}(s-2)\right) \cong H^{1}\left(Z, O_{Z}(t-2)\right) \cong H^{1}\left(C, O_{C}(t+s-2)\right)=0
$$

$(C$ is a complete intersection of surfaces of degrees $t$ and $s), H^{1}\left(X, O_{X}(t+s-2)\right.$ ) is zero too. Now we can apply Proposition 2.1: $X$ is smoothable.

REMARK 3.3. This procedure was applied in [BM] in order to construct for every $n$ a smooth minimal curve with Hartshorne-Rao module concentrated in one degree and of dimension $n$, minimal (in various senses) in its liaison class. This was performed by induction, "adding" at every step a pair of skew lines, and then smoothing the reducible curve thus obtained.

Let us call $Y_{n}$ this curve, and let $Z$ be a smooth maximal rank sextic curve of genus 2 or 1 . It is contained in a smooth surface $Q_{4}$ of degree 4 . It is possible to add by liaison $Y_{n}$ and $Z$ by means of $Q_{4}$ and of a smooth surface of degree $2 n$ containing $Y_{n}$, and then to apply the previous proposition. The result is a smooth connected curve of degree $2 n^{2}+8 n+6$.

4. Applications to nodal unions of curves. Other applications of our general statement can be found in smoothing nodal unions of curves; in particular we can prove the following proposition (where the roles of $Y$ and $Z$ are not symmetric).

PROPOSITION 4.1. Let $Y$ and $Z$ be smooth curves with transversal intersection, $X=Y \cup Z, D=Y \cap Z$. Suppose that $H^{0}\left(X, O_{X}(n)\right) \rightarrow H^{0}\left(Z, O_{Z}(n)\right)$ is surjective, that there exists a section $\xi \in H^{0}\left(X, \omega_{X}(-n)_{\mid Y}\right)$ different from zero on $D$ and that $h^{1}\left(\mathrm{P}_{k}^{3}, I_{X}(n+3)\right)=0=h^{2}\left(\mathrm{P}_{k}^{3}, I_{X}(n+2)\right)$. Then it is possible to apply to $X$ Theorem 2.1.

ProOF. With these hypotheses, it is enough to find $\varphi \in H^{0}\left(X, \omega_{X}(-n)\right)$, different from zero on $\operatorname{Sing} X=D$. But it is possible to prove (as in Theorem 3.2) that the restriction map $\delta: H^{0}\left(X, \omega_{X}(-n)\right) \rightarrow H^{0}\left(X, \omega_{X}(-n)_{\mid Y}\right)$ is surjective; so we can choose a section $\varphi \in H^{0}\left(X, \omega_{X}(-n)\right)$ such that $\delta(\varphi)=\xi$.

A first application of this result is in smoothing curves with a line attached at $p$ points; this problem was studied (with different techniques) in [HH and BE]. 
COROLLARY 4.2. Let $Y$ be a smooth connected curve such that

$$
h^{1}\left(\mathrm{P}_{k}^{3}, I_{Y}(p+1)\right)=0=h^{1}\left(\mathrm{P}_{k}^{3}, I_{Y}(p-2)\right), \quad h^{1}\left(Y, O_{Y}(p)\right)=0,
$$

$Z$ a line meeting $Y$ transversally in $p$ points, and $Y \cup Z=X$ such that

$$
h^{0}\left(\mathrm{P}_{k}^{3}, I_{X}(p+1)\right)=h^{0}\left(\mathrm{P}_{k}^{3}, I_{Y}(p+1)\right)-2 .
$$

Then $X$ is smoothable (in the sense of Theorem 2.1).

ProOF. Let as usual $D=Y \cap Z$. We prove that $Y$ and $Z$ satisfy the hypothesis of 4.1 , with $n=p-2$. Since

$\omega_{X}(2-p)_{\mid Z} \cong \omega_{Z}(2-p) \otimes O_{Z}(D) \cong O_{Z}(2-p-2) \otimes O_{Z}(p) \cong O_{Z}(2-p-2+p) \cong O_{Z}$,

we have a section of this sheaf different from zero on $D$. On the other hand, the restriction map $\rho: H^{0}\left(\mathrm{P}_{k}^{3}, \mathrm{O}_{\mathrm{P}^{3}}(p-2)\right) \rightarrow H^{0}\left(Y, \mathrm{O}_{Y}(p-2)\right)$ is surjective $(0=$ $\left.h^{1}\left(\mathrm{P}_{k}^{3}, I_{Y}(p-2)\right)\right)$, and therefore the restriction map

$$
H^{0}\left(X, O_{X}(p-2)\right) \rightarrow H^{0}\left(Y, O_{Y}(p-2)\right)
$$

too. So it is enough to show that $h^{1}\left(X, O_{X}(p)\right)$ and $h^{1}\left(\mathrm{P}_{k}^{3}, I_{X}(p+1)\right)$ are trivial. But there is an exact sequence

$$
0 \rightarrow O_{X}(p) \rightarrow O_{Y}(p) \oplus O_{Z}(p) \rightarrow O_{D}(p) \rightarrow 0
$$

from which we get a long exact sequence

$$
\begin{aligned}
\rightarrow H^{0}\left(Y, O_{Y}(p)\right) \oplus H^{0}\left(Z, O_{Z}(p)\right) \rightarrow H^{0}\left(D, O_{D}(p)\right) \\
\rightarrow H^{1}\left(X, O_{X}(p)\right) \rightarrow H^{1}\left(Y, O_{Y}(p)\right) \rightarrow 0,
\end{aligned}
$$

where the map $H^{0}\left(Y, O_{Y}(p)\right) \oplus H^{0}\left(Z, O_{Z}(p)\right) \rightarrow H^{0}\left(D, O_{D}(p)\right)$ is surjective, since already the natural restriction $H^{0}\left(Z, O_{Z}(p)\right) \rightarrow H^{0}\left(D, O_{D}(p)\right)$ is surjective $(\# D=$ $p)$. On the other hand, $H^{1}\left(Y, O_{Y}(p)\right)=0$ by hypothesis, and therefore $H^{1}\left(X, O_{X}(p)\right)$ $=0$. Thanks to the hypothesis $h^{0}\left(\mathrm{P}_{k}^{3}, I_{X}(p+1)\right)=h^{0}\left(\mathrm{P}_{k}^{3}, I_{Y}(p+1)\right)-2$, a simple dimensional computation in the exact sequence

$$
\begin{gathered}
0 \rightarrow H^{0}\left(\mathrm{P}_{k}^{3}, I_{X}(p+1)\right) \rightarrow H^{0}\left(\mathrm{P}_{k}^{3}, I_{Y}(p+1)\right) \oplus H^{0}\left(\mathrm{P}_{k}^{3}, I_{Z}(p+1)\right) \\
\rightarrow H^{0}\left(\mathrm{P}_{k}^{3}, I_{D}(p+1)\right) \rightarrow H^{1}\left(\mathrm{P}_{k}^{3}, I_{X}(p+1)\right) \rightarrow 0
\end{gathered}
$$

shows that $h^{1}\left(\mathrm{P}_{k}^{3}, I_{X}(p+1)\right)=0$. Then $X$ is smoothable.

REMARK 4.3. The hypothesis $h^{0}\left(\mathrm{P}_{k}^{3}, I_{X}(p+1)\right)=h^{0}\left(\mathrm{P}_{k}^{3}, I_{Y}(p+1)\right)-2$ is satisfied if, for instance, $Y$ is contained in only one surface $Q$ of degree $p$, the only surfaces of degree $p+1$ containing $Y$ are of the form $Q \cup H$, where $H$ is a plane, and $Z$ is a line not lying on $Q$.

EXAMPLE 4.4. If $Y$ is a nonspecial smooth connected curve with $h^{1}\left(\mathrm{P}_{k}^{3}, I_{Y}(2)\right)=0$, then we can apply the previous corollary with $p=1$.

Of course, if $p>1$ the applicability of this corollary is wider. For instance, a smooth curve of degree 7 and genus 6 (that is a curve of type $(4,3)$ on a smooth quadric surface) with a secant line in two points is always smoothable.

EXAMPLE 4.5. Let $Y$ be a plane quartic and $C$ a conic meeting $Y$ in two points. Then $X=Y \cup C$ is smoothable (this was already proved in [HH]). In fact, let $C$ be a conic; $\omega_{C} \cong O_{C}(-1)$, and $\omega_{X \mid C} \cong O_{C}$. We therefore have a nonzero section of $\omega_{X \mid C}$, and $H^{0}\left(X, O_{X}\right) \rightarrow H^{0}\left(Y, O_{Y}\right)$ is surjective. But also the map 
$H^{0}\left(Y, O_{Y}(2)\right) \oplus H^{0}\left(C, O_{C}(2)\right) \rightarrow H^{0}\left(D, O_{D}(2)\right)$ is surjective (here $\left.D=Y \cap C\right)$. Therefore, $h^{1}\left(Y, O_{Y}(2)\right)=h^{1}\left(X, O_{X}(2)\right)=0$.

Let us now consider the exact sequence

$$
\begin{aligned}
0 & \rightarrow H^{0}\left(\mathrm{P}_{k}^{3}, I_{X}(3)\right) \rightarrow H^{0}\left(\mathrm{P}_{k}^{3}, I_{Y}(3)\right) \oplus H^{0}\left(\mathrm{P}_{k}^{3}, I_{C}(3)\right) \\
& \rightarrow H^{0}\left(\mathrm{P}_{k}^{3}, I_{D}(3)\right) \rightarrow H^{1}\left(\mathrm{P}_{k}^{3}, I_{X}(3)\right) \rightarrow 0 .
\end{aligned}
$$

It is easy to compute that $h^{0}\left(\mathrm{P}_{k}^{3}, I_{D}(3)\right)=18, h^{0}\left(\mathrm{P}_{k}^{3}, I_{C}(3)\right)=13, h^{0}\left(\mathrm{P}_{k}^{3}, I_{Y}(3)\right)=$ 10 , and $h^{0}\left(\mathrm{P}_{k}^{3}, I_{X}(3)\right)=5$, and therefore $h^{1}\left(\mathrm{P}_{k}^{3}, I_{X}(3)\right)=0$. Therefore we can apply Proposition 4.1 .

PROPOSITION 4.6 ("SMOOTHING BASIC DOUBLE LINKAGES"). Let $Y$ be a smooth connected strongly $k$-regular curve and let $S$ be a smooth surface of degree $p \leq k+1$ containing $Y$. Let $Q_{s}$ be a general smooth surface of degree $s$, and $Z=S \cap Q_{s}$. Then $X=Y \cup Z$ is smoothable.

PROOF. There is a nontrival $\xi$ section of

$$
\begin{aligned}
H^{0}\left(X, \omega_{X}(-k-s+4)_{\mid Y}\right) & \cong H^{0}\left(Y, \omega_{Y}(-k-s+4) \otimes O_{Y}(s)\right) \\
& \cong H^{0}\left(Y, \omega_{Y}(-k+4)\right) \cong H^{1}\left(Y, O_{Y}(k-4)\right) \neq 0 .
\end{aligned}
$$

If $Q_{s}$ is general, $Z=S \cap Q_{s}$ is smooth and cuts transversally $Y$ in points where $\xi$ does not vanish. Now we try to apply Proposition 4.1, with $n=k+s-4$. In fact, $\rho: H^{0}\left(\mathrm{P}_{k}^{3}, O_{\mathrm{P}^{3}}(k+s-4)\right) \rightarrow H^{0}\left(Z, O_{Z}(k+s-4)\right)$ is surjective (since $Z$ is a complete intersection), and therefore also the restriction map

$$
H^{0}\left(X, O_{X}(k+s-4)\right) \rightarrow H^{0}\left(Z, O_{Z}(k+s-4)\right)
$$

is surjective. As usual, it is enough now to show that $h^{1}\left(X, O_{X}(k+s-2)\right)$ and $h^{1}\left(\mathrm{P}_{k}^{3}, I_{X}(k+s-1)\right)$ are trivial.

Let us denote by $M(T)$ the Hartshorne-Rao module of a (not necessarily smooth) curve $T$, that is $M(T)=\bigoplus_{n} H^{1}\left(\mathrm{P}_{k}^{3}, I_{T}(n)\right)$. Thanks to the results of $[\mathbf{S C H}]$, $M(X)$

$\cong M(Y)(s)$, and therefore $h^{1}\left(\mathrm{P}_{k}^{3}, I_{X}(k+s-1)\right)=h^{1}\left(\mathrm{P}_{k}^{3}, I_{Y}(t k-1)\right)=0$.

In order to show that $h^{1}\left(X, O_{X}(k+s-2)\right)=0$, we make use of the exact sequence

$$
0 \rightarrow O_{Y}(-s) \rightarrow O_{X} \rightarrow O_{Z} \rightarrow 0
$$

in the associated long exact sequence we have

$$
\rightarrow H^{1}\left(Y, O_{Y}(k-2)\right) \rightarrow H^{1}\left(X, O_{X}(k+s-2)\right) \rightarrow H^{1}\left(Z, O_{Z}(k+s-2)\right) \rightarrow \cdots .
$$

On the other hand $H^{1}\left(Z, O_{Z}(k+s-2)\right)=H^{2}\left(\mathrm{P}_{k}^{3}, I_{Y}(k-2)\right)=0$, if $k+s-2-p-s \geq$ -3 , that is if $p \leq t k+1$. This completes the proof.

EXAMPLE 4.7. An arithmetically normal curve with a complete intersection attached (of the previous kind) is smoothable. Remark that, thanks to [GP], every projective normal reduced and irreducible curve is smoothable.

More generally, let

$$
v(Y)=\max \left\{n \mid h^{1}\left(\mathrm{P}_{k}^{3}, I_{Y}(n)\right) \neq 0\right\}
$$

and

$$
e(Y)=\max \left\{n \mid h^{1}\left(Y, O_{Y}(n)\right) \neq 0\right\}
$$


and suppose $v(Y) \leq e(Y)+2$. Then there actually exist smooth surfaces of degree $p=e(Y)+4$ or $e(Y)+5$ containing $Y$, and therefore it is possible to apply Proposition 4.6.

If we consider the union of the smooth sextic curves of genus 1 or 2 of Remark 3.3 with the intersection of $Q_{4}$ and a general surface of every degree, it is smoothable.

\section{REFERENCES}

[BE] E. Ballico and Ph. Ellia, Note to the paper "On degeneration of projective curves", Thèse, Nice, 1983.

[BM] G. Bolondi and J. C. Migliore, Classification of maximal rank curves in the liaison, classes $L_{n}$, Math. Ann. 277 (1987), 585-603.

[CO] I. Coanda, The Chern classes of the stable rank 3 vector bundles on $\mathrm{P}^{3}$, Math. Ann. 273 (1985), 65-79.

[GLP] L. Gruson, R. Lazarsfeld and Ch. Peskine, On a theorem of Castelnuovo, and the equations defining space curves, Invent. Math. 72 (1983), 491-506.

[GP] L. Gruson and Ch. Peskine, Genre des courbes de l'espace projective. I, Algebraic Geometry (Tromsø, 1977), Lecture Notes in Math., vol. 687, Springer-Verlag, Berlin and New York, 1978, pp. 31-59.

[HA] R. Hartshorne, Stable reflexive sheaves, Math. Ann. 254 (1980), 165-190.

[HH] R. Hartshorne and A. Hirschowitz, Smoothing algebraic space curves, Algebraic Geometry, Sitges (Barcelona, 1983), Lecture Notes in Math., vol. 1126, Springer-Verlag, Berlin and New York, 1985, pp. 98-113.

[HII] A. Hirschowitz, Sur la postulation générique des courbes rationelles, Acta Math. 146 (1981), 209-230.

[HI2] - Existence de faisceaux réfléxifs de rang deux sur $\mathrm{P}^{3}$ à bonne cohomologie, Prepublications Mathématiques de l'Université de Nice, 1986, no. 116.

[LR] R. Lazarsfeld and P. Rao, Linkage of general curves of large degree, Algebraic GeometryOpen Problems (Ravello, 1982), Lecture Notes in Math., vol. 997, Springer-Verlag, Berlin and New York, 1983, pp. 267-289.

[SCH] Ph. Schwartau, Liaison addition and monomial ideals, Ph. D. Thesis, Brandeis University, 1982.

[SER] E. Sernesi, On the existence on certain families of curves, Invent. Math. 26 (1974), 271-302. ITALY

Dipartimento di Matematica, Università di Trento, I-38050 Povo (Trento),

Current address: Dipartimento di Matematica e Fisica, Università di Camerino, I-62032 Camerino (Macerata), Italy 